\title{
SPLINE INTERPOLATION AND BEST QUADRATURE FORMULAE
}

\author{
BY I. J. SCHOENBERG \\ Communicated by Felix Browder, October 9, 1963
}

1. The spline interpolation formula. A spline function $S(x)$, of degree $k(\geqq 0)$, having the knots

$$
x_{0}<x_{1}<\cdots<x_{n}
$$

is by definition a function of the class $C^{k-1}$ which reduces to a polynomial of degree not exceeding $k$ in each of the $n+2$ intervals in which the points (1) divide the real axis. The function $S(x)$ is seen to depend linearly on $n+k+1$ parameters. In [5, Theorem 2, p. 258] are given the precise conditions under which we can interpolate uniquely by $S(x)$ arbitrarily given ordinates at $n+k+1$ points on the real axis.

For the remainder of this note we set $k=2 m-1(1 \leqq m \leqq n)$ and single out from this family of spline functions the

Class $\Sigma_{m}$ : The class of spline functions $S(x)$ of degree $2 m-1$, knots (1), and the additional property that $S(x)$ reduces to polynomials of degree not exceeding $m-1$ in each of the ranges $\left(-\infty, x_{0}\right)$ and $\left(x_{n},+\infty\right)$.

The restriction that $m \leqq n$ is essential, otherwise $\Sigma_{m}$ reduces to $\pi_{m-1}$ (here and below $\pi_{k}$ denotes a generic polynomial of degree $\leqq k$, as well as their class). In a paper [1] soon to appear C. de Boor observes that [5, Theorem 2] implies the following interesting

Theorem 1 (C. DE BooR). Given $m(1 \leqq m \leqq n)$, the points (1) and also arbitrary reals $y_{i}(i=0, \cdots, n)$, then there is a unique $S(x)$ such that

$$
S\left(x_{i}\right)=y_{i} \quad(i=0, \cdots, n) .
$$

Let us now consider this interpolating spline function $S(x)$ in a given finite interval $a \leqq x \leqq b$ containing the points (1). Its particular interest is due to the following

ThEOREM 2 (C. DE BOOR). Let $f(x) \in C^{m-1}[a, b]$, having an absolutely continuous $f^{(m-1)}(x)$, and be such that

$$
f\left(x_{i}\right)=y_{i} \quad(i=0, \cdots, n) .
$$

If $S(x)$ denotes the interpolating spline function of Theorem 1 then

$$
\int_{a}^{b}\left(f^{(m)}(x)\right)^{2} d x \geqq \int_{a}^{b}\left(S^{(m)}(x)\right)^{2} d x
$$


with equality if and only if $f(x)=S(x) .^{1}$

In other words: The interpolating $S(x) \in \Sigma_{m}$ minimizes the integral $\int_{a}^{b}\left(y^{(m)}\right)^{2} d x$ among all interpolating functions and in this sense $S(x)$ is the best interpolating function for the order $m$. For $m=1 S(x)$ reduces to the linear interpolation between consecutive points while $S(x)=y_{0}$ if $x \leqq x_{0}$ and $S(x)=y_{n}$ if $x \geqq x_{n}$. For $m=2$ these results are due to Holladay [2]. The periodic analogue for $m=2$ was found by Walsh, Ahlberg and Nilson [8] and for an arbitrary $m$ see [9]. The results of [1] and [9] were also found independently, but later (September 1963), by the author (see [7]). Holladay and de Boor consider only the case when $a=x_{0}, x_{n}=b$.

We may express the interpolating $S(x)$ of Theorem 1 in the form

$$
S(x)=\sum_{0}^{n} y_{\nu} L_{\nu}(x)
$$

where $L_{v}(x)$ are the fundamental functions, i.e. $L_{i}\left(x_{j}\right)=\delta_{i j}$. The function $f(x)$ being defined in $[a, b]$ we consider the interpolation formula (I. F.)

$$
f(x)=\sum_{0}^{n} f\left(x_{\nu}\right) L_{\nu}(x)+R(x ; f)
$$

which we call the spline I. F. of order $m(m \leqq n)$. Observe that $R\left(x ; \pi_{m-1}\right)=0$.

2. Best quadrature formulae. By integrating (3) over the range $[a, b]$ and setting

$$
A_{\nu}=\int_{a}^{b} L_{\nu}(x) d x
$$

we obtain the quadrature formula (Q.F.)

$$
\int_{a}^{b} f(x) d x=\sum_{0}^{n} A_{v} f\left(x_{v}\right)+R_{A}(f)
$$

which is evidently also exact if $f \in \pi_{m-1}$, i.e. $R_{A}\left(\pi_{m-1}\right)=0$.

${ }^{1}$ As a matter of fact the interpolating spline $S(x)$ of Theorem 1 enjoys yet another fundamental minimal property to be found in [7, Theorem 1]. It may be stated as follows: Let $f(x)$ be defined as in Theorem 2 and let $S(x)$ be the unique element of $\Sigma_{m}$ interpolating $f(x)$ at the points (1). Then $S(x)$ is also uniquely characterized, up to an additive $\pi_{m-1}$ by the condition: $\left({ }^{*}\right)$ To minimize $\int_{a}^{b}\left(S^{(m)}(x)-f^{(m)}(x)\right)^{2} d x$ for $S(x) \in \Sigma_{m}$. In [7] Theorems 1 and 2 are derived from the minimal property $\left(^{*}\right)$ of $S(x)$. The property $\left(^{*}\right)$ was discovered by Walsh, Ahlberg and Nilson [8] for the periodic case of cubic splines $(m=2)$ and apparently now generalized by them to all $m$ in the periodic case (see [9]). 
Concerning quadrature formulae A. Sard [4] proposed in 1949 the following very reasonable scheme: Let $1 \leqq m \leqq n$ and let

$$
\int_{a}^{b} f(x) d x=\sum_{0}^{n} B_{v} f\left(x_{\nu}\right)+R_{B}(f)
$$

be a $Q . F$. which is exact if $f(x) \in \pi_{m-1}$. This requirement is equivalent to the relations

$$
\frac{b^{r+1}-a^{r+1}}{r+1}=\sum_{\nu=0}^{n} B_{\nu} x_{\nu}^{r} \quad(r=0, \cdots, m-1),
$$

which still leave $n-m+1$ free parameters. These are disposed of as follows: By Peano's theorem [3] we can write

$$
R_{B}(f)=\frac{1}{(m-1) !} \int_{a}^{b} K(x) f^{(m)}(x) d x
$$

and we now determine the $B_{\nu}$ by the requirement that

$$
\int_{a}^{b}(K(x))^{2} d x=\text { minimum, }
$$

with the side-conditions (7). This method leads to a $Q$. F. (6) which Sard very appropriately calls the Best $Q$. F. for the given $x_{\nu}$, given $[a, b]$ and given order $m$.

Sard computes the coefficients $B_{\nu}$ of (6), for equidistant $x_{\nu}$ at unit steps, $(a, b)=\left(x_{0}, x_{n}\right)$ and $m=1,2,3,4$ and $n=1, \cdots, 6(m \leqq n)$. Holladay [2] computes the coefficients $A_{\nu}$ of (5), for $m=2$, equidistant $x_{\nu},(a, b)=\left(x_{0}, x_{n}\right)$ and $n=2,3, \cdots, 10$. On comparing their tables it is seen that Holladay's formulae are identical with Sard's for $m=2 .^{2}$

That these are no mere numerical accidents is shown by

Theorem 3. The Q. F. (5), obtained by integrating the spline I. F. (3), is identical with Sard's Best Q. F. (6).

The key to the proof is the following remark: The I. F. (3) is not only exact if $f \in \pi_{m-1}$ but also if $f \in \Sigma_{m}$, because of the unicity in Theorem 1. Therefore also the $Q . F$. (5) is exact if $f \in \Sigma_{m}$. Conversely, this property uniquely characterizes (5), for if $f(x)=L_{\nu}(x)$ and $R_{A}\left(L_{v}\right)=0$, then (5) implies (4).

The identity of (5) with (6) will therefore follow as soon as we have established the following

2 The author is here performing the meritorious service of decreasing the number of known $Q$. F. by showing that two known sets are identical. 
Lemma. Sard's $Q . F .(6)$ is exact if $f \in \Sigma_{m}$.

Proof. We assume the relations (7) and then by Peano's theorem

$$
\begin{aligned}
K(x)=\frac{1}{m}(b-x)_{+}^{m}-\frac{1}{m}(a-x)_{+}^{m}-\sum_{0}^{n} B_{\nu}\left(x_{\nu}-x\right)_{+}^{m-1} & \\
& (-\infty<x<\infty),
\end{aligned}
$$

where we use the function $y_{+}=(y+|y|) / 2$. We now solve the problem (9), (7) by Lagrange's multiplier method and consider the expression

$$
E=\frac{1}{2} \int_{a}^{b}(K(x))^{2} d x+\sum_{r=0}^{m-1} \lambda_{r}\left(\sum_{\nu=0}^{n} B_{\nu} x_{\nu}^{r}-\left(b^{r+1}-a^{r+1}\right)(r+1)^{-1}\right) .
$$

Its partial derivatives $\partial E / \partial B_{i}$ furnish the $n+1$ relations

$$
\begin{aligned}
\int_{a}^{b}\left\{-\frac{1}{m}(b-x)^{m}+\sum_{0}^{n} B_{\nu}\left(x_{\nu}-x\right)_{+}^{m-1}\right\}\left(x_{i}-x\right)_{+}^{m-1} d x & \\
+\sum_{r=0}^{m-1} \lambda_{r} x_{i}^{r} & =0 \quad(i=0, \cdots, n),
\end{aligned}
$$

which are satisfied by the coefficients $B_{\nu}$ of (6) together with appropriate constants $\lambda_{r}$.

As a preliminary step towards the proof of the Lemma let us evaluate the integral appearing in (10) and show that (10) may also be written in the equivalent form

$$
\begin{aligned}
-\frac{1}{2 m}\left(x_{i}-a\right)^{2 m}+\sum_{\nu=0}^{n} B_{v}\left(x_{i}-x_{v}\right)_{+}^{2 m-1}+\sum_{r=0}^{m-1} \lambda_{r}^{\prime} x_{i}^{r} & =0 \\
(i & =0, \cdots, n),
\end{aligned}
$$

where the $\lambda_{r}^{\prime}$ are proportional to the $\lambda_{r}$. To this end we observe that (7) are equivalent to the identity

$$
\frac{1}{m}(a-x)^{m}-\frac{1}{m}(b-x)^{m}+\sum_{0}^{n} B_{\nu}\left(x_{\nu}-x\right)^{m-1}=0 .
$$

Subtracting this vanishing expression within the curly brackets in (10) and using the identity $y_{+}^{m-1}-y^{m-1}=(-1)^{m}(-y)_{+}^{m-1}$, we may write (10) as

$$
\begin{array}{r}
\left(10^{\prime}\right) \int_{a}^{b}\left\{-\frac{1}{m}(a-x)^{m}+(-1)^{m} \sum_{0}^{n} B_{\nu}\left(x-x_{\nu}\right)_{+}^{m-1}\right\} \\
\left(x_{i}-x\right)_{+}^{m-1} d x \\
+\sum_{r=0}^{m-1} \lambda_{r} x_{i}^{r}=0 .
\end{array}
$$


Now the integrals can be evaluated explicitly:

$$
\begin{gathered}
\int_{a}^{b}(a-x)^{m}\left(x_{i}-x\right)_{+}^{m-1} d x=(-1)^{m} \gamma_{0} \cdot\left(x_{i}-a\right)^{2 m}, \\
\int_{a}^{b}\left(x-x_{\nu}\right)^{m-1}\left(x_{i}-x\right)_{+}^{m-1} d x=\gamma_{1} \cdot\left(x_{i}-x_{\nu}\right)_{+}^{2 m-1}
\end{gathered}
$$

where

$$
\gamma_{0}=\int_{0}^{1} t^{m}(i-t)^{m-1} d t, \quad \gamma_{1}=\int_{0}^{1} t^{m-1}(1-t)^{m-1} d t .
$$

Observing that $\gamma_{1}=2 \gamma_{0}$ and substituting the values just found into $\left(10^{\prime}\right)$, we see that it reduces to (11) where $\lambda_{r}^{\prime}=(-1)^{m} \lambda_{r} /\left(2 \gamma_{0}\right)$.

A proof of the Lemma now follows easily. Indeed, the most general $S \in \Sigma_{m}$ has the form

(12) $S(x)=\sum_{0}^{n} C_{i}\left(x_{i}-x\right)_{+}^{2 m-1}+P_{m-1}(x)\left(C_{i}\right.$ constants, $\left.P_{m-1} \in \pi_{m-1}\right)$

in which the condition that $S(x) \in \pi_{m-1}$ in $(-\infty, x)$ requires the relations

$$
\sum_{i=0}^{n} C_{i} x_{i}^{r}=0 \quad(r=0, \cdots, m-1)
$$

But then

$$
R_{B}(S)=\sum C_{i} R_{B}\left(\left(x_{i}-x\right)_{+}^{2 m-1}\right)+R_{B}\left(P_{m-1}\right)
$$

or, since the last term vanishes,

$$
R_{B}(S)=\sum_{0}^{n} C_{i} \frac{1}{2 m}\left(x_{i}-a\right)^{2 m}-\sum_{i=0}^{n} C_{i} \sum_{\nu=0}^{n} B_{\nu}\left(x_{i}-x_{\nu}\right)_{+}^{2 m-1} .
$$

But the right-hand side expression vanishes: Indeed, we obtain it by multiplying the left side of (11) by $-C_{i}$ and summing over $i$ and then take into account the relations (13). Thus $R_{B}(S)=0$ and the Lemma is established.

3. Concluding remarks. Let me point out again, as I already did in [6], that the connection between the problems of interpolation and of mechanical quadratures becomes closer and more illuminating if we interpolate not only by polynomials but by the wider class of splien functions. The Q.F. (6) was derived by a method entirely foreign 
to interpolation and yet, as seen, it arises also from the I. F. (3) by integration. There are many other similar examples.

\section{REFERENCES}

1. C. de Boor, Best approximation properties of spline functions of odd degree, J. Math. Mech. 12 (1963), 747-749.

2. J. C. Holladay, Smoothest curve approximation, Math. Tables Aids Comput. 11 (1957), 233-243.

3. G. Peano, Residuo in formulas de quadratura, Mathesis 34 (1914), 1-10.

4. A. Sard, Best approximate integration formulas; best approximation formulas, Amer. J. Math. 71 (1949), 80-91.

5. I. J. Schoenberg and Anne Whitney, On Polya frequency functions. III. The positivity of translation determinants with an application to the interpolation by spline curves, Trans. Amer. Math. Soc. 74 (1953), 246-259.

6. I. J. Schoenberg, Spline functions, convex curves and mechanical quadratures, Bull. Amer. Math. Soc. 64 (1958), pp. 352-357.

7. - On interpolation by spline functions and its minimal properties, Proceedings of the Conference on Approximation, Oberwolfach, Germany, August 4-10, 1963. (to appear)

8. J. L. Walsh, J. H. Ahlberg and E. N. Nilson, Best approximation properties of the spline fit, J. Math. Mech. 11 (1962), 225-234.

9. - Best approximation and convergence properties of higher-order spline fits, Abstract 63t-103, Notices Amer. Math. Soc. 10 (1963), 202.

University of Pennsylvania and INSTITUTE FOR ADVANCED STUDY 